\title{
A Computational Study of Mother Rotor VF in the Human Ventricles.
}

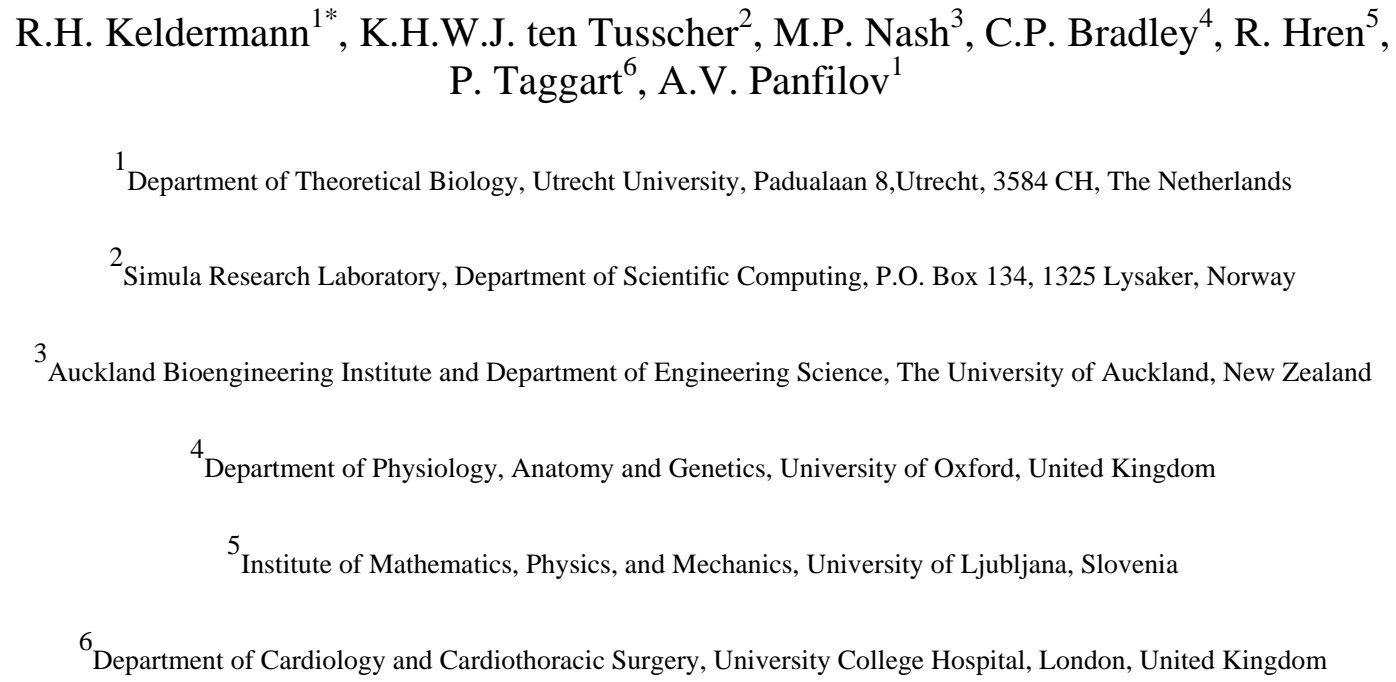

Running head: A computational study of mother rotor VF

Contact information: Address for reprint requests and other correspondence: R.H.Keldermann, Utrecht University, Department of Theoretical Biology, Padualaan 8, 3584 CH Utrecht, The Netherlands, telephone:+0031(0)302531497 fax:+0031(0)30-2513655 e-mail: R.H.Keldermann@uu.nl

*Author to whom correspondence should be addressed. Email: R.H.Keldermann@uu.nl Tel: +31 302531497 


\begin{abstract}
Sudden cardiac death is one of the major causes of death in the industrialized world. It is most often caused by a cardiac arrhythmia called ventricular fibrillation (VF). Despite its large social and economical impact, the mechanisms for VF in the human heart yet remain to be identified. Two of the most frequently discussed mechanisms observed in experiments with animal hearts are the multiple wavelet and the mother rotor hypotheses. Most recordings of $\mathrm{VF}$ in animal hearts are consistent with the multiple wavelet mechanism. However, in animal hearts, mother rotor fibrillation has also been observed. For both multiple wavelet and mother rotor VF, cardiac heterogeneity plays an important role.

Clinical data of action potential restitution measured from the surface of human hearts were recently published. These in vivo data show a substantial degree of spatial heterogeneity. Using these clinical restitution data, we study the dynamics of VF in the human heart using a heterogeneous computational model of the human ventricles. We hypothesized that this observed heterogeneity can serve as a substrate for mother rotor fibrillation.

We found that, based on these data, mother rotor VF can occur in the human heart and that ablation of the mother rotor terminates VF. Furthermore, we found that both mother rotor and multiple wavelet VF can occur in the same heart depending on the initial conditions at the onset of VF. We studied the organization of these two types of VF in terms of filament numbers, excitation periods and frequency domains.

We conclude that mother rotor fibrillation is a possible mechanism in the human heart.
\end{abstract}

Keywords: action potential duration-restitution-heterogeneity; reentrant arrhythmias; ventricular fibrillation; computer simulation. 


\section{Introduction}

Sudden cardiac death is the most common cause of death in the industrialized world and in most cases it is due to ventricular fibrillation (VF) [62]. During VF, excitation waves are disturbed causing the contraction of the ventricles to become rapid and uncoordinated. It has been shown in clinical and experimental studies that these turbulent wave patterns are underpinned by re-entrant sources of excitation [6, 11, 56, 28, 15, 52]. If VF is not halted by means of defibrillation, this condition will be lethal within several minutes.

Several mechanisms have been proposed to explain the dynamics of VF. One widely studied mechanism is known as multiple wavelet VF [26, 27] and is characterized by the presence of multiple self-sustained electrical wavelets in the heart. These wavelets may be arise due to dynamical instabilities, which are associated with action potential duration (APD) restitution properties (the so called restitution hypothesis) [34, 12, 36, 54, 40, 9, 37] and intracellular calcium dynamics [55]

Another well-established mechanism is known as mother rotor fibrillation, in which VF is driven by a dominant fast source of excitation [17, 2, 61]. This rapid reentrant electrical source (i.e., the mother rotor) is responsible for maintaining VF and causes conduction block in the surrounding tissue due to heterogeneity in refractory periods [1]. The activation source is so fast that it is unable to conduct in some regions where it blocks but manages to conduct in other regions where refractoriness allows. As a result, multiple small wavebreaks and irregular activation patterns are generated [17].

Mother rotors have been observed in VF experiments in animal hearts. Samie et al. [44] reported the presence of a high frequency rotor in the left ventricle responsible for maintaining VF in guinea pig hearts. They also demonstrated that a spatial left-right gradient in provides a robust ionic mechanism for rotor stabilization in the left ventricle and wavebreak generation in the right ventricle. Mother rotor fibrillation has also been reported in rabbit hearts [2, 57]. Furthermore, long-lived, stationary rotors have been reported in isolated right ventricular slaps of sheep [61]. Multiple attempts to find similar mother rotor VF in the pig heart were not successful [16, 21, 20]. Although these studies showed the presence of long lasting epicardial rotors, they were not consistently present throughout VF. The authors concluded that epicardial mother rotors did not drive VF in their experimental model, however the presence of a transmural mother rotor could not be ruled out. In the human heart, several clinical studies have investigated VF organization on the epicardial and/or endocardial surface [31, 33, 30, 25]. In particular, the study by Nash et al. [33] reported persistent epicardial rotors that lasted over 5 seconds, corresponding to 20 reentrant cycles or more. However, similar to studies on pig hearts [16, 21, 20], these rotors were not consistently present throughout the measured VF episodes. The study by Masse et al. [25] showed an example of a persistent rotor on the endocardium co-existing with multiple reentrant patterns occurring on the epicardium, and suggested that this could be a recording of mother rotor fibrillation. However, as in the study of Nash et al. [33] this persistent rotor was not consistently present throughout the measured VF episode. Finally both these studies [33, 25] could not determine whether these long lasting rotors were responsible for driving VF or represented only part of the multiple wavelet activity.

One factor contributing to the plausibility of mother rotor VF is electrophysiological tissue heterogeneity [61]. In heterogeneous tissue the period of rotation varies spatially, and if a rotor is placed in a region where rotation is fastest, then it may serve as a mother rotor that drives VF [44]. Recently, in clinical studies [60, 32], large degree of electrophysiological tissue heterogeneity were also reported in human hearts.

The primary aim of this paper was to study whether the electrophysiological tissue heterogeneity recorded by Nash et al. [32] could provide a substrate to support mother rotor VF in the human heart. We define mother rotor VF as a stationary persistent source that lasted for at least 5 seconds and actively induced wavebreaks. In addition, elimination of the mother rotor must result in VF termination. Having identified such rotors, we also studied the organization of the resulting mother rotor VF and compared this with multiple wavelet VF in the human heart.

This study utilized an integrative model of the human ventricles, which incorporates a detailed ionic model of the human ventricular myocyte [51,50], combined with an anatomically realistic geometry of the human ventricles, which includes fiber direction anisotropy [14]. Recently, this model was extended [23] to include heterogeneous APD restitution data from the human ventricles obtained from [32]. Our modeling framework allows us to check whether VF in the human heart can be driven by a mother rotor, and to study how properties of the mother rotor (such as its frequency) affect the VF excitation patterns. Performing such studies experimentally is difficult, if not impossible. 


\section{Materials and Methods}

We use a realistic model of the human ventricles, which contains a detailed description of cell electrophysiology, ventricular anatomy and fiber direction anisotropy [51,50]. We have extended this model to include clinically measured restitution data of the human ventricles [32] using our previously developed diffusion based algorithm [23].

\section{Human Ventricular Model}

We model excitable behavior using a monodomain description of cardiac tissue [22]:

$$
C_{m} \frac{\partial V_{m}}{\partial t}-\frac{\partial}{\partial x_{i}}\left(D_{i j} \frac{\partial V_{m}}{\partial x_{j}}\right)-I_{i o n}
$$

where $V_{m}$ denotes the transmembrane voltage, $C_{m}$ the membrane capacitance, $D_{i j}$ the diffusion tensor accounting for the anisotropy of cardiac tissue, and $\mathrm{I}_{\text {inn }}$ the sum of the ionic transmembrane currents describing the excitable behavior of the individual ventricular cells. To represent human ventricular electrophysiological properties we used the ionic model developed by Ten Tusscher et al. [50] and refer to this as the TNNP model. This model provides a detailed description of voltage, ionic currents and intracellular ion concentrations. The model represents the intracellular calcium dynamics by including subspace calcium dynamics, which controls the L-type calcium current and the calcium-induced calcium release (CICR). CICR is modeled with a four-state Markov model for the ryanodine receptor, and both the fast and slow voltage-gated inactivation of the L-type calcium current are also incorporated (see [50] for detailed information). The model reliably reproduces experimentally measured APD [29, $32]$ and conduction velocity restitution curves [10].

Geometric data describing the 3D ventricular anatomy and fiber direction field are derived from a normal healthy human heart [14] and is described in more detail in [49]. Overall, the heart was represented by approximately 13.5 million points with a spatial resolution of $0.25 \mathrm{~mm}$. As in [49], we assume that the transverse conductivity is the same in all directions orthogonal to the longitudinal direction of the muscle fiber axis and that local conductivity tensors can be derived from local muscle fiber directions using:

$$
D_{i j}=D_{L} * \delta_{i, j}+\left(D_{L}-D_{T}\right) \alpha_{i} \alpha_{j}
$$

where, $\mathrm{D}_{\mathrm{L}}$ and $\mathrm{D}_{\mathrm{T}}$ are the longitudinal and transverse conductivity, respectively and $\alpha$ is the muscle fiber direction. For $\mathrm{D}_{\mathrm{L}}$ we used $162 \Omega \mathrm{cm}$ and for $\mathrm{D}_{\mathrm{T}}$ we used $40.5 \Omega \mathrm{cm}$, which resulted in conduction velocities of 68 $\mathrm{cm} / \mathrm{s}$ in the longitudinal direction and $32 \mathrm{~cm} / \mathrm{s}$ in the transverse direction, similar to the values reported by [48]. The resulting anisotropy ratio was approximately 2:1, which is also consistent with clinical measurements [19].

\section{Clinical Data on Restitution Heterogeneity}

We have used clinical restitution data from [32], which provided global epicardial APD restitution properties in cardiac patients undergoing aortic valve replacement or coronary artery bypass graft procedures. Activation-recovery intervals (ARI) were recorded on the entire ventricular epicardial surface using an epicardial sock containing 256 unipolar contact electrodes (inter electrode spacing $\sim 10 \mathrm{~mm}$ ). ARI values have been shown to correlate with the $50 \%$ action potential duration repolarization values $\left(\mathrm{APD}_{50}\right)$ [13]. In each electrode, restitution properties were determined using a standard S1-S2 protocol. Then, clinical restitution data was fitted using a least-squares mono-exponential fit, and maximum restitution slopes $\left(\mathrm{S}_{\max }\right)$ were determined for each electrode. For more information about the clinical data and procedures used we refer to [32].

For this study, we used data from a single patient. This dataset contains a large degree of heterogeneity and steep slopes of measured APD restitution (see Supplementary Material, Figure 1). The mean \pm SD $S_{\max }$ was $2.01 \pm 1.20$ based on $n=243$ (95\%) valid restitution curves (see [23]). We assigned these restitution properties throughout the entire 3D ventricular mass. However, data on the full 3D organization of APD restitution in the human ventricles are presently not available. Observations have been reported only for surfaces of the heart. To date, either epicardial or endocardial recordings for individual hearts have been reported [32,60]. For both the epicardial and endocardial 
data, the maximum restitution slopes varied markedly between regions and the spatial arrangement of these regions varied considerably within and between patients. It is likely that this heterogeneity has a 3D structure.

In the absence of a 3D restitution profile, we extrapolated the epicardial data from Nash et al. [32] across the 3D mass. To this end, we developed a diffusion based algorithm to smoothly extend the epicardial profile across the ventricular muscle. We first extended the 243 clinically measured surface $S_{\max }$ values across the entire ventricular walls by solving a diffusion problem. The variable $S_{\max }$ was kept constant at each electrode position during the diffusion process. As a result, we obtained a smooth spatial interpolation of $S_{\max }$ across the ventricles, except for regions immediately adjacent to the recording electrodes. The second step was to remove these sharp gradients by applying a $3 \times 3 \times 3$ voxel moving average window. The third step involved a linear scaling to correct all 13.5 million $\mathrm{S}_{\max }$ values based on the original known $243 \mathrm{~S}_{\max }$ values at the recording electrode locations. For a more extensive description of the diffusion algorithm, we refer to [23]. We believe that this is a reasonable first step to qualitatively describe APD restitution heterogeneity until more detailed data on the 3D organization of restitution in the human restitution becomes available.

The results of this interpolation for the current dataset are shown in Figure 1 (polar plots shown in Supplementary Materials, Figure 1). We see that a large region of the posterior wall contains steep restitution slopes, with maximum slope values up to 3 . The left ventricle contains more shallow restitution slopes, with maximum slope values of approximately $0.5-1$. Between these regions, intermediate values are present. The mean \pm SD restitution slope is $2.01 \pm 0.25$ ( $n=13.5$ million).

\section{Numerical Approach}

Equations for the gating variables in the TNNP model were integrated using a Rush and Larsen integration scheme [43]. To integrate Equation 1, we used a forward Euler scheme with a time step of $\Delta t=0.02 \mathrm{~ms}$ and a space step of $\Delta x=0.25 \mathrm{~mm}$. No-flux boundary conditions were used so that the axial current flow from heart points to nonheart points was set zero. For more details we refer to [49, 23]

\section{Induction of Ventricular Fibrillation}

To initiate 3D scroll waves, we used a S1-S2 protocol, for which a S1 stimulus was applied at either the right ventricles or on the posterior junction of the right and left ventricles. The S2 stimulus was activated during the refractory tail of the S1 stimulus and was extended from the base for approximately $50 \%$ of the base-apex dimension, thereby creating a single scroll wave. We varied the position of the S2 stimulus such that the core of the initial scroll wave was located in different regions of the ventricles, with either shallow or steep restitution curves (see Figure 1). Stimulus currents were applied at twice the diastolic threshold value. We performed different simulations of VF by initiating the scroll wave at different locations (see Figure 1, black and white circles). The difference between these black and white circles will be described later. Furthermore, we also initiated a scroll wave in the septum (not shown in Figure 1). Simulations were run for 8 seconds or were terminated if there was no activity present.

\section{Electrograms}

Assuming that the medium is an infinite volume conductor, electrograms can be calculated using the dipole source density of the membrane potential in all voxel points using [38]:

\section{$E C G=\int_{V} \frac{D W_{m} \cdot t}{r^{2}} d V$}

where, $V$ is the domain of integration (i.e., the ventricular volume), and $\vec{r}$ is the vector from each point to the recording electrode, which was placed $10 \mathrm{~cm}$ from the center of the ventricles in the anterior direction of the transverse plane.

\section{Filaments and Phase Singularities}

Scroll wave filaments were detected using an algorithm proposed by Fenton and Karma [8]. If there is a spiral wave in 2D (a scroll wave in 3D) present, the core (filament) can be defined as the point(s) for which the excitation 
wavefront and waveback meet. This can be calculated as the intersection points of an isopotential line (we used -60 $m V$ ) and the $d V / d t=0$ iso-line. Voxel data corresponding to these intersection points were then stored. Individual filaments were detected by iteratively joining neighboring voxels that were designated as filament points. Filaments were determined at $10 \mathrm{~ms}$ intervals. Of these filaments, we tracked: the time of birth, time of death, time of bifurcation, time of amalgamation, lifespan, filament from which a filament bifurcated, filament into which a filament amalgamated, and ultimate filament to which a filament can be traced back through the bifurcations events [49]. Filaments were categorized based on their geometrical position and assigned to either the left ventricle, right ventricle or septum.

\section{Period Distribution and Frequency Domains}

For all simulations we calculated the interbeat interval between successive action potentials in every heart point ( $n=13.5$ million points) following the initiation of VF (i.e., after the first 2 seconds of simulation time). For the histograms (Figures 5 and 6) the mean period was calculated in every point between 2-6 seconds of simulation time. The histogram range is $0.16-0.28$ seconds and the bin size $1 \mathrm{~ms}$. Note that this method is different from experimental methods which calculate the dominant frequency via fast Fourier transformations of optical action potential mappings [61, 44, 57, 33]. Because experimental recordings are often measured during time intervals which exceed our simulation time (minutes vs seconds) we prefer to use direct measurement of period values.

\section{Implementation}

All simulations were coded in C++ and MPI and were run on 16 processors of a Beowulf cluster consisting of 16 Dell 650 Precision Workstations (dual Intel Xeon $2.66 \mathrm{GHz}$ ). Simulating 1 second of wave propagation in the ventricles took approximately 6.5 hours of wall-clock computation time. Ventricular geometry, wave patterns and scroll wave filaments were visualized using the marching cubes algorithms for isosurface detection in voxel data, and OpenGL for isosurface rendering.

\section{Results}

\section{Multiple Wavelet and Mother Rotor VF Wavefronts}

Scroll waves were initiated at different locations of the ventricles (see Figure 1, white and black circles). After investigating the results of the different starting locations, we found that we could distinguish two different types of VF dynamics which were dependent on the location of the first spiral.

The first type of VF dynamics we found is multiple wavelet VF and was investigated in our previous publication [23]. The starting locations of the initial spiral for this type of VF are denoted by the black circles in Figure 1. These initial locations were located in regions containing steep restitution slopes. Figure 2 shows a typical example of these dynamics. After initiation, we observed that the spiral wave broke up into multiple wavelets that were predominantly located in the right ventricle, where APD restitution was steepest (slopes of 2.5-3). The chaotic spatio-temporal patterns that arose persisted throughout the simulations and were similar to the restitution induced multiple wavelet VF dynamics we studied in [49, 23].

The second type of VF we found is mother rotor VF. For several other initial conditions (denoted by the white circles in Figure 1), located in regions with shallow restitution slopes, we found patterns of excitation that were different from the patterns described above. In both cases the mother rotor was similar in size. Figure 3 shows an example of such an excitation pattern. After an initial transient, we observed a persistent (single) stable spiral wave located in the left ventricle, where restitution slopes were shallow (slopes of 0.5-1.0). At the same time, we observed the continuous formation of wavebreaks occurring on the other side of the heart (the free wall of the right ventricle and posterior wall), where restitution slopes were steep (slopes of 2.5-3). These wave patterns are consistent with mother rotor fibrillation. In order to verify this, we show below that these wavebreaks were indeed driven by the single stable spiral in the left ventricle.

During mother rotor VF wavebreaks were induced by dynamical heterogeneity (due to steep slopes of the APD restitution curves). Indeed, we found that wavebreaks occurred not immediately, but after several cycles of mother rotor rotation. Moreover, APD alternans instability was clearly evident at the locations of onset of wave breaks. This wavebreak mechanism differs from the mechanism of wavebreaks due to conduction blocks between regions with different refractoriness that is usually reported to underlie mother rotor fibrillation. 


\section{Ablation of the Mother Rotor}

To test the hypothesis that the wavebreaks observed in Figure 3 are driven by the mother rotor, we eliminated the mother rotor spiral 3 seconds after its onset by removing approximately $20 \%$ of the left ventricular free wall of the left ventricle that contained this rotor (see Figure 4). We found that after removal, the wavebreaks did not complete rotation and that the wavetips of the rotors ran into the refractory tails of the waves and disappeared. After 0.5 seconds there was no wave activity present in the heart. Thus, the wavebreaks in the right ventricle (Figure 3) seem to be driven by the stable spiral wave in the left ventricle implying that this is indeed mother rotor fibrillation. We tested this removal procedure further by applying it at different phases of the mother rotor rotation, and by removing different sized regions (10-20\% of the LV free wall). We found that as soon as the mother rotor activity was eliminated, the wavebreaks in the right ventricle died out.

Clearly, removing part of the myocardium decreased the overall ventricular mass of the heart, which could potentially terminate wave activity during fibrillation (critical mass hypothesis). To verify that this was not the case here, we repeated the multiple wavelet simulations shown in Figure 2 and removed the same part of the left ventricle as we did for the mother rotor simulation. We found that multiple wavelet VF did not terminate after the removal of tissue, and that the right ventricle sustained multiple independent sources, which could complete a rotation and were not terminated by the tissue removal procedure (see Supplementary Material, Figure 2). Thus termination of mother rotor VF was not related to the critical mass phenomenon, but to the removal of a persistent rotor that was responsible for actively inducing wavebreaks.

These results demonstrate that VF in Figure 3 is driven by a single spiral and represents mother rotor VF. Interestingly, our simulations show that multiple wavelet and mother rotor VF can occur in the same heart, depending on the initial location of the spiral.

\section{VF Dynamics and Organization}

\section{ECG, Period Distribution and Frequency Domains}

In Figure 5 we show the ECG and period distributions for both the multiple wavelet and mother rotor simulations. For multiple wavelet VF (Figure 5A) the ECG had a mean frequency of 4.4-4.5 Hz, similar to clinically recorded frequencies [4, 31, 33, 59]. For the mother rotor fibrillation (Figure 5B) the mean frequency was very similar and had a mean frequency of 4.6-4.7 Hz. Both ECGs have similar complexity. Note, that the shape of the FFT spectra for the ECGs shown in Figure 5A,B were similar (see Supplementary Materials, Figure 7), and there did not appear to be any relationship between period distribution and underlying heterogeneity profile (see Supplementary Materials, Figure 6).

We also determined period values for every point ( $n=13.5$ million heart points) from 2 to 6 seconds and constructed a histogram of mean period values for these simulations (Figure 5C,D). We see that histograms for the mother rotor and for the multiple wavelet VF differ substantially. For multiple wavelet VF (Figure 5C) we see a broad period distribution with mean period of $0.223(4.48 \mathrm{~Hz})$ (box-plot stats: median $=0.217$, interquartilerange $=0.016, n=13.5$ million), while for the mother rotor VF (Figure 5D) the distribution has a clear peak at 0.216 seconds, corresponding to the period of the mother rotor, and a narrow distribution. Overall the mean \pm was 0.216 (4.63 Hz)(box-plot stats: median=0.216, interquartile-range $=0.002, n=13.5$ million).

In Figure 3 of the Supplementary Materials, we show the corresponding 3D spatial distribution of the mean period values for the multiple wavelet and mother rotor simulation measured between 2-4 and 4-6 seconds, respectively. Indeed, during multiple wavelet VF period values are grouped into different domains with different frequencies (Supplementary Materials, Figure 3A). However, these domains are not anatomically pre-defined but change during the course of the simulation (Supplementary Materials, Figure 3B) as they are determined by dynamical processes [23]. For the mother rotor simulations, we did not observe distinct frequency domains for the durations between 2-4 (Supplementary Materials, Figure 3C) or 4-6 seconds. In this case, the period distribution was mainly determined by the frequency of the mother rotor.

\section{Number of Filaments}

A convenient way to quantify the complexity of excitation patterns in the ventricles of the heart is by determining the number of the excitation sources (scroll wave filaments) [49, 52, 42, 41, 21, 3, 5, 35]. A scroll wave filament is a line around which a 3D spiral wave rotates. When such a filament intersects with the surface of the heart, a phase singularity (PS) manifests on the heart surface. In Figure 4 of the Supplementary Material, we compare the time dynamics of the total number of filaments and epicardial PSs for both the multiple wavelet and the mother rotor simulation.

For the multiple wavelet simulation, the number of filaments varied between 10-30. The mean number of filaments was 11.2 and the mean number of PS was 8.0. Initially there were only filaments detected in the right 
ventricle, but after approximately 4.5 seconds of simulation time there were also filaments detected in the left ventricle. Most wavebreaks were found in high restitution areas, but sometimes wavebreaks also occurred in areas with shallow restitution slopes. Additional characteristics of multiple wavelet VF organization such as location of breaks have been previously discussed in [23]. The ratio of the number of filaments to PSs was approximately 1.4, consistent with earlier findings [49].

For the mother rotor VF, the number of filaments varied between 2-8, which is lower compared to multiple wavelet VF. The mean number of filaments was 3.8 and the mean number of PS was 2.3, resulting in a 1.65 ratio between filaments and epicardial PS. Apart from the mother rotor filament in the left ventricle, most filaments occurred in the right ventricle.

\section{Filament History}

We also analyzed filament history by tracing the events of filament creation and elimination over 8 seconds of time through death, birth, bifurcation and amalgamation events. This is shown in Figure 5 of the Supplementary Materials, for both the multiple wavelet and the mother rotor fibrillation simulations.

For the multiple wavelet VF simulation, we detected 1025 filaments. Most of the filaments existed only for short periods of time ( $<0.5$ seconds). However, we also observed few long living filaments, which lasted for several cycles (up to 2-3 seconds). The mean lifespan of a filament was 0.084 seconds $(n=1025)$.

For the mother rotor fibrillation simulation, we detected 388 filaments. During this simulation the mother rotor filament was stable and was present until the end of the simulation. All other filaments existed for short periods of time $(<0.25-0.5$ seconds). Similar results have been reported for rabbit hearts where VF was driven by a mother rotor [2]. The mean lifespan of a filament was 0.068 seconds ( $n=387$, mother rotor filament excluded).

We find that the total number of filaments during mother rotor fibrillation is 2-3 times smaller compared to the multiple wavelet fibrillation. In addition, during mother rotor fibrillation filaments have a shorter lifetime $(p<0.05$, unpaired Student t-test). Furthermore, during multiple wavelet VF there were several filaments that lasted longer (up to 2-3 seconds), while during mother rotor fibrillation only short lived filaments accompanied the (long-lasting) mother rotor. Similar results were found for the simulations using other initial conditions that resulted in either multiple wavelet or mother rotor VF (see Table 1).

\section{Effect of Mother Rotor Frequency on VF Dynamics}

If fibrillation is driven by a mother rotor, then influencing the frequency of the mother rotor should have a substantial effect on VF dynamics. We performed additional simulations in which we decreased the maximal conductance of the L-type Ca current in the region in which the mother rotor resided to 50, 35 and 25\% of it's original value. This intervention increased the frequency of the mother rotor and resulted in flattening of the restitution slope in the region of the mother rotor. This did not change the type of rotation of the mother rotor, which remained stable after the intervention.

The results of these simulations are shown in Figure 6. Figure 6A shows the same period distribution as in Figure $5 \mathrm{D}$ for normal $\mathrm{I}_{\mathrm{CaL}}$ conductance. We see a narrow unimodal period distribution with a mean period of 0.216 seconds. Spatially distinct frequency domains were not observed (see Supplementary Materials, Figure 3C).

We see that for a $50 \% \mathrm{I}_{\mathrm{CaL}}$ reduction the mean period of VF decreased to 0.204 seconds (see Figure 6B). We also see that the period distribution became wider and that a small second peak arose around a period of 0.218 sec. When we decreased $\mathrm{I}_{\mathrm{CaL}}$ to $35 \%$ of its normal conductance a bimodal period distribution arose (see Figure 6C), with a first peak around an mean period of $0.202 \mathrm{sec}$. and a second peak with a mean period of $0.210 \mathrm{sec}$. When we decreased $\mathrm{I}_{\mathrm{CaL}}$ to $25 \%$ of its original conductance, the split in the period distribution became more pronounced (see Figure 6D). The first peak had a mean period value of 0.197 sec., whereas the second peak increased to a mean of 0.252 sec., which is much larger than the mean period value for the original settings of $(0.216 \mathrm{sec})$.

The bimodal period distributions that occur for decreased $\mathrm{I}_{\mathrm{CaL}}$ conductance reflects an underlying $3 \mathrm{D}$ spatial distribution of periods, as is shown in Figure 7A,B for the case were $\mathrm{I}_{\mathrm{CaL}}$ is reduced to $25 \%$ of its original value. We clearly see that the heart is subdivided into different frequency domains: the mother rotor area (which has a period of 0.197 sec. (accounting for the left peak in Figure 6D)), and the area around the right ventricle where most of the wavebreaks occur and which has a much longer period of excitation (accounting for the right peak in Figure 6D). Such domains of different frequency of excitation are similar to those reported in experimental studies of atrial and ventricular mother rotor fibrillation [61, 18]. These domains arise when differences in refractory periods between different regions are large enough to cause Wenckebach like conduction blocks, which is presumed to be the sole mechanism for wave break formation during mother rotor type fibrillation.

We conclude that increasing the frequency of the mother rotor changes the process of the onset of breaks during VF. For normal $\mathrm{I}_{\mathrm{CaL}}$ the wavebreaks are caused by dynamical instability: the first breaks occur only after a number of rotations of the initial spiral and the onset of these breaks was clearly associated with alternans in APD. This 
indicates that steep APD restitution mediated alternans was the mechanism for wave break formation. However, since these wavebreaks only occurred in steep regions far away from the initial spiral core, they led to a mother rotor type rather than a multiple wavelet type of VF. When $\mathrm{I}_{\mathrm{CaL}}$ was decreased to more than $50 \%$, breaks occured straight after the formation of the initial spiral without any alternans (which is an important characteristic for wavebreaks caused by dynamical heterogeneity). This is because the frequency of the mother rotor increased so much that differences in refractory period lead to local conduction block as was also reported in [24]. Thus, anatomical (and not dynamical) heterogeneity was responsible for wavebreak formation.

In addition, we found that due to the increased frequency of the mother rotor the total number of filaments produced during 8 seconds increased from 388 ( $\mathrm{I}_{\mathrm{caL}}$ times 1.0) to 538 ( $\mathrm{I}_{\mathrm{caL}}$ times 0.25 ). Thus, speeding up of the mother rotor not only changes the mechanism of wave break formation but also increases the complexity of VF.

\section{Discussion}

In this study, we report on mother rotor fibrillation in an electrophysiologically heterogeneous computational model of the human ventricles. We defined a mother rotor as a stationary, persistent source that actively drives VF, which terminates when the mother rotor is eliminated. However, it is also possible that a source driving VF is transient. We also call this a mother rotor if VF can be stopped by the removal of this source. It is understood that there may be special cases for which multiple wavelet VF can be eliminated by the removal of a single source. However, we think that in most cases true mother rotor VF will be correctly classified using this definition.

It should be noted that mother rotor like VF can occur without tissue substrate heterogeneity. Fenton and Cherry [7] showed that mother rotor like excitation patterns can occur as a result of discordant alternans-induced breakup far from the center of the spiral. In addition, rotors that anchor to inexcitable obstacles [45, 57, 53] (such as papillary muscles, fibrosis) may also result in mother rotor like VF. Furthermore, additional sources of heterogeneity (such as increased fibrosis) can lead to local conduction block and development of reentry, and are also important for the initiation and organization of VF [58].

Our study shows that increased heterogeneity in the human heart can result in the onset of mother rotor VF. However, it should be emphasized that any type of heterogeneity, i.e. from local dynamic or fixed heterogeneities (including APD restitution), favors the formation of local wavebreaks and thus provides a substrate to sustain VF of any type (e.g. multiple wavelets) and not exclusively mother rotor VF.

Although our model is based on clinical data on heterogeneity and involves a detailed description of the structure of the heart and properties of cardiac cells, our conclusions should be further tested in experimental and clinical studies involving high spatial resolution recordings of wave activity in the human heart during VF.

In our model, we consistently reproduced mother rotor VF if the initial spiral wave was located in the left ventricle where APD was shortest and APD restitution shallowest. We found multiple wavelet VF when the initial spiral was located in the right ventricle where APD restitution was steep and APD was longer. In our model, shallow restitution curves were correlated with shorter APDs and periods, whereas steep restitution curves corresponded to longer APDs and periods (see [23]). Theoretically, it should be possible to have a model in which longer periods correspond to steeper restitution curves. However, in such a case we believe mother rotor fibrillation would not occur, since a rotor in a region of steep restitution would be unstable and would therefore lead to multiple wavelet type VF.

To demonstrate that the mother rotor was indeed responsible for driving the wavebreaks, we mimicked local ablation to remove the mother rotor spiral. After removing the tissue that supported the mother rotor, we found that all wavebreaks ended and the fibrillation was terminated within 0.5 seconds of simulation time. However, we do not think that local ablation of a mother rotor is of clinical use, as multiple mechanisms of VF can occur in the same heart depending on the initiation procedure. However, we cannot exclude the possibility that local interventions may be used to stop a specific episode of VF. Note that if the mother rotor is terminated by other means, e.g. it slows down and is overrun by other wavelets, then VF is not necessarily terminated.

We then analyzed the differences in the underlying dynamics between mother rotor and multiple wavelet fibrillation. ECG signals for mother rotor and multiple wavelet VF were similar and resembled clinically observed ECG signals during VF. During multiple wavelet VF, we observed a broad period distribution that was mainly determined by dynamical processes, rather than underlying heterogeneity (see also [23]). In contrast, during mother rotor VF we observed that the periods were dominated by the period of the mother rotor, leading to a narrow distribution. Apart from the mother rotor, only short lived wave breaks were present during mother rotor fibrillation, whereas during multiple wavelet VF several long lived filaments were present. In addition, during mother rotor VF, approximately 2-3 times less filaments were present compared to multiple wavelet VF. This difference is due to the fact that during mother rotor fibrillation the fast mother rotor dominates a large part of the cardiac tissue. Hence, 
other wavebreaks did not have enough time nor space to independently persist.

For the mother rotor fibrillation with the default $\mathrm{I}_{\mathrm{CaL}}$ settings, we found that wavebreaks in the right ventricle occurred due to dynamical instabilities caused by steep APD restitution slopes (i.e., alternans). Wavebreaks occurred after several cycles of mother rotor rotation and at places where the breaks occurred there was alternans instability in APD. Excitation periods across the entire tissue were dictated by the mother rotor, and different frequency domains were not present.

We found that decreasing the period of the mother rotor (by decreasing the $\mathrm{I}_{\mathrm{CaL}}$ conductance in the mother rotor region), and thus increasing anatomical heterogeneity, leads to Wenckebach like conduction blocks due to large differences in refractory period. Wavebreaks now occurred immediately after initiation of the mother rotor and were anatomically predefined. Consequently, different frequency domains were present, similar to experimental findings for mother rotor type VF [61].

We thus have shown that the formation of domains of different frequency is dependent on the frequency of the mother rotor and the degree of anatomical heterogeneity in refractory periods. Therefore, mother rotor type fibrillation is not necessarily associated with different frequency domains.

For multiple wavelet fibrillation, flattening restitution slope has been proposed as a promising therapeutic intervention [9]. However, for mother rotor fibrillation, besides absolute restitution slopes, heterogeneity in restitution slopes and APD is a major determinant for VF occurrence. This implies that reducing electrophysiological heterogeneity may also be a fruitful target for therapeutic intervention.

\section{Limitations}

Despite the fact that our model incorporates a detailed description of the electrophysiological properties of human ventricular cells, a realistic human ventricular geometry including fiber direction field, and a detailed description of ventricular electrophysiological heterogeneity, there are number of shortcomings of our study.

A first limitation is that clinical data were only available for the epicardial surface of the ventricles. There was no information on restitution properties in the mid-myocardium nor endocardium of these hearts. As a consequence, the epicardial restitution slope data had to be extrapolated across the entire ventricular walls using a diffusion-based algorithm as reported in [23]. The use of different parameters in our diffusion algorithm may lead to different gradients in slope values for the same set of clinical data. It remains to be investigated how these different heterogeneity profiles with different transmural slope gradients affect the results presented in this study.

Second, other heterogeneities, such as epi-, endocardial, and M-cells, Purkinje fibers, laminar sheets, and disease conditions (such as fibrosis and gap junction remodeling), were not taken into account in our model. Additional heterogeneities may further contribute to the complexity of excitation patterns during VF.

A third limitation to our study is that the ionic mechanisms responsible for the variation in APD restitution are presently unknown. It should be noted that the mapping of the TNNP model parameters to the restitution slope values that we used is not unique and that the same slopes may likely be obtained using other combinations of parameter settings. However, we believe that this non-uniqueness should not qualitatively affect the results of our study as long as shallow restitution curves correspond to shorter periods, and steep restitution curves correspond to longer periods.

Finally, the mechanisms of VF studied in this paper are not the only mechanisms that may potentially cause VF. As an example, apart from steep APD restitution, other non-linear instabilities, e.g. in the intracellular calcium dynamics [39, 46, 47], may also lead to alternans and VF.

\section{Conclusions}

In this paper, we report the finding of mother rotor VF in a computational model of the human ventricles. Furthermore, we showed that mother rotor and multiple wavelet VF can occur in the same heart depending on the initial location of reentry. We show that the underlying VF dynamics is markedly different between mother rotor and multiple wavelet VF. Mother rotor VF was more organized, with a smaller number of filaments and a more uniform distribution of rotor periods. Ablation of the mother rotor was sufficient to terminate VF. Studying the basic mechanisms responsible for maintaining and terminating VF in the human heart is important for understanding the pathogenesis of sudden cardiac death.

\section{Acknowledgments}

We would like to thank Prof. D.J. Paterson and the Wellcome Trust for assistance with the human heart APD restitution data. 


\section{Grants}

This research was funded by the Netherlands Organization for Scientific Research (NWO grant number 814.02.014). M.P.N. acknowledges previous support from the Marsden Fund Council from New Zealand government funding, administered by the Royal Society of New Zealand.

\section{Disclosures}

None.

\section{References}

[1] A. Baher, Z. Qu, A. Hayatdavoudi, S. T. Lamp, M. J. Yang, F. Xie, S. Turner, A. Garfinkel, and J. N. Weiss. Short-term cardiac memory and mother rotor fibrillation. Am. J. Physiol. Heart. Circ. Physiol., 292(1):H180H189, January 2007.

[2] J. Chen, R. Mandapati, O. Berenfeld, A. C. Skanes, and J. Jalife. High-frequency periodic sources underlie ventricular fibrillation in the isolated rabbit heart. Circ. Res., 86(1):86-93, January 2000.

[3] R.H. Clayton and A.V. Holden. A method to quantify the dynamics and complexity of re-entry in computational models of ventricular fibrillation. Phys. Med. Biol., 47:225-238, 2002.

[4] R.H. Clayton, A. Murray, and R.W. Campbell. Objective features of the surface electrocardiogram during ventricular tachyarrhythmias. Eur. Heart J., 16:1115-1119, 1995.

[5] R.H. Clayton, E.A. Zhuchkova, and A.V. Panfilov. Phase singularities and filaments: Simplifying complexity in computational models of ventricular fibrillation. Prog Biophys Mol Biol., 90:378-398, 2006.

[6] J.M. Davidenko, A.M. Pertsov, R. Salomonsz, W. Baxter, and J. Jalife. Stationary and drifting spiral waves of excitation in isolated cardiac muscle. Nature, 355:349-351, 1992.

[7] F. Fenton, E.M. Cherry, H.M. Hastings, and S.J. Evans. Multiple mechanisms of spiral wave breakup in a model of cardiac electrical activity. Chaos, 12:852-892, 2002.

[8] F. Fenton and A. Karma. Vortex dynamics in three-dimensional continuous myocardium with fiber rotation: filament instability and fibrillation. Chaos, 8:20-47, 1998.

[9] A. Garfinkel, Y. H. Kim, O. Voroshilovsky, Z. Qu, J. R. Kil, M. H. Lee, H. S. Karagueuzian, J. N. Weiss, and P. S. Chen. Preventing ventricular fibrillation by flattening cardiac restitution. Proc. Natl. Acad. Sci. U.S.A., 97(11):6061-6066, May 2000.

[10] S.D. Girouard, J.M. Pastore, K.R. Laurita, K.W. Gregory, and D.S. Rosenbaum. Optical mapping in a new guinea pig model of ventricular tachycardia reveals mechanisms for multiple wavelengths in a single reentrant circuit. Circulation, 93:603-613, 1996.

[11] R.A. Gray, A.M. Pertsov, and J. Jalife. Spatial and temporal organization during cardiac fibrillation. Nature, 392:75-78, 1998.

[12] M.R. Guevara, A. Ward, A. Shrier, and L. Glass. Electrical alternans and period doubling bifurcations. IEEE Comp. Cardiol., 562:167-170, 1984.

[13] C.W. Haws and R.L Lux. Correlation between in vivo transmembrane action potential durations and activation-recovery intervals from electrograms. Effects of interventions that alter repolarization time. Circulation, 81(1):281-88, 1990.

[14] R. Hren. A realistic model of the human ventricular myocardium: Application of the study of ectopic activation. $\mathrm{PhD}$ thesis, Dalhouse University, Halifax, 1996.

[15] J. Huang, J.M. Rogers, C.R. Killingsworth, K.P. Sting, W.M. Smith, and R. Ideker. Evolution of activation patterns during long-duration ventricular fibrillation in dogs. Am J Physiol Heart Circ Physiol., 101:H11391200, 2004.

[16] J. Huang, G. P. Walcott, C. R. Killingsworth, S. B. Melnick, J. M. Rogers, and R. E. Ideker. Quantification of activation patterns during ventricular fibrillation in open-chest porcine left ventricle and septum. Heart. Rhythm., 2(7):720-728, July 2005.

[17] J. Jalife. Ventricular fibrillation: mechanisms of initiation and maintenance. Annual Rev. Physiol., 62:25-50, 2000.

[18] J. Jalife, O. Berenfeld, and M. Mansour. Mother rotors and fibrillatory conduction: a mechanism of atrial fibrillation. Cardiovasc. Res., 54(2):204-216, May 2002.

[19] H.J. Jongsma and R. Wilders. Gap junctions in cardiovascular disease. Circ. Res., 86:1193-1197, 2000.

[20] M. W. Kay and J. M. Rogers. Epicardial rotors in panoramic optical maps of fibrillating swine ventricles. Conf. Proc. IEEE. Eng. Med. Biol. Soc., 1:2268-2271, 2006.

[21] M.W. Kay, G.P. Walcott, J.D. Gladden, S.B. Melnick, and J.M. Rogers. Lifetimes of epicardial rotors in 
panoramic optical maps of fibrillating swine ventricles. Am. J. Physiol., 291(4):H1935-41, 2006.

[22] J. Keener and J. Sneyd. Mathematical physiology. Springer-Verlag, New York, Heidelberg, Berlin, 1998.

[23] R. H. Keldermann, K. H. Ten Tusscher, M. P. Nash, R. Hren, P. Taggart, and A. V. Panfilov. Effect of heterogeneous APD restitution on VF organization in a model of the human ventricles. Am. J. Physiol. Heart. Circ. Physiol., 294(2):H764-H774, February 2008.

[24] V. I. Krinsky. Spread of excitation in an inhomogeneous medium (state similar to cardiac fibrillation). Biophysics, 11:776-784, 1966.

[25] S. Masse, E. Downar, V. Chauhan, E. Sevaptsidis, and K. Nanthakumar. Ventricular fibrillation in myopathic human hearts: mechanistic insights from in vivo global endocardial and epicardial mapping. Am. J. Physiol. Heart. Circ. Physiol., 292(6):H2589-H2597, June 2007.

[26] G. K. Moe. On the multiple wavelet hypothesis of atrial fibrillation. Arch. Int. Pharmacodyn. Ther., 140:183188, 1962.

[27] G. K. Moe, W. C. Rheinbolt, and J. A. Abildskov. A computer model of atrial fibrillation. Am. Heart J., 67:200-220, 1964.

[28] J. Moreno, A.V. Zaitsev, M. Warren, O. Berenfeld, J. Kalifa, E. Lucca, S. Mironov, Guha. P., and J. Jalife. Effect of remodelling, stretch and ischaemia on ventricular fibrillation frequency and dynamics in a heart failure model. J. Cardiovas. Res., 65:158-166, 2005.

[29] J.M. Morgan, D. Cunningham, and E. Rowland. Dispersion of monophasic action potential duration: demonstrable in humans after premature ventricular extrastimulation but not in steady state. J. Am. Coll. Cardiol., 19:1244-1253, 1992.

[30] K. Nanthakumar, J. Jalife, S. Masse, E. Downar, M. Pop, J. Asta, H. Ross, V. Rao, S. Mironov, E. Sevaptsidis, J. Rogers, G. Wright, and R. Dhopeshwarkar. Optical mapping of Langendorff-perfused human hearts: establishing a model for the study of ventricular fibrillation in humans. Am. J. Physiol. Heart. Circ. Physiol., 293(1):H875-H880, July 2007.

[31] K. Nanthakumar, G.P. Walcott, S. Melnick, J.M. Rogers, M.W. Kay, W.M. Smith, R.E. Ideker, and W. Holman. Epicardial organization of human ventricular fibrillation. Heart Rhythm, 1:14-23, 2004.

[32] M.P. Nash, C.P. Bradley, P.M. Sutton, R.H. Clayton, P. Kallis, M.P. Hayward, D.J. Paterson, and P. Taggart. Whole heart action potential duration restitution properties in cardiac patients: a combined clinical and modelling study. Exp. Physiol., 91(2):339-54, 2006.

[33] M.P. Nash, A. Mourad, R.H. Clayton, P.M. Sutton, C.P. Bradley, M. Hayward, D.J. Paterson, and P. Taggart. Evidence for multiple mechanisms in human ventricular fibrillation. Circulation, 114(6):536-42, 2006.

[34] J.B. Nolasco and R.W. Dahlen. A graphic method for the study of alternation in cardiac action potentials. J. Appl. Physiol., 25:191-196, 1968.

[35] A.V. Panfilov. Three-dimensional organization of electrical turbulence in the heart. Phys. Rev. E, 59:R6251R6254, 1999.

[36] A.V. Panfilov and A.V. Holden. Self-generation of turbulent vortices in a two-dimensional model of cardiac tissue. Phys. Lett. A, 147:463-466, 1990.

[37] A.V. Panfilov and A. M. Pertsov. Ventricular fibrillation: evolution of the multiple wavelet hypothesis. Phil. Trans. R. Soc. Lond. A., 359:1315-1325, 2001.

[38] R. Plonsey and R.C. Barr. Bioelectricity. Plenum Publishing Corporation, NY, 1989.

[39] R.P. Pruvot, E.J. Katra, D.S. Rosenbaum, and K.R. Kaurita. Role of calcium cycling versus restitution in the mechanism of repolarization alternans. Circ. Res., 94:1083-1090, 2004.

[40] Z. Qu, J.N. Weiss, and A. Garfinkel. Cardiac electrical restitution properties and stability of reentrant spiral waves: a simulation study. Am. J. Physiol., 276:H269-H283, 1999.

[41] J. Rogers. Combined phase singularity and wavefront analysis for optical maps of ventricular fibrillation. IEEE Trans. Biomed. Eng., 92:56-65, 2004.

[42] J. Rogers, J. Huang, W.M. Smith, and R.E. Ideker. Incidence, evolution and spatial distribution of functional reentry during ventricular fibrillation in pigs. Circ. Res., 84:945-954, 1999.

[43] S. Rush and H. Larsen. A practical algorithm for solving dynamic membrane equations. IEEE Trans. Biomed. Eng., 25:389-392, 1978.

[44] F. H. Samie, O. Berenfeld, J. Anumonwo, S. F. Mironov, S. Udassi, J. Beaumont, S. Taffet, A. M. Pertsov, and J. Jalife. Rectification of the background potassium current: a determinant of rotor dynamics in ventricular fibrillation. Circ. Res., 89:1216-1223, 2001.

[45] F. H. Samie and J. Jalife. Mechanisms underlying ventricular tachycardia and its transition to ventricular fibrillation in the structurally normal heart. Cardiovasc. Res., 50(2):242-250, May 2001.

[46] Y. Shiferaw, M.A. Watanabe, A. Garfinkel, J.N. Weiss, and A. Karma. Model of intracellular calcium cycling 
in ventricular myocytes. Biophys. J., 85:3666-3686, 2003.

[47] W. Shimizu and C. Antzelevitch. Cellular and ionic basis for T-wave alternans under long-QT conditions. Circulation., 99:1499-1507, 1999.

[48] P. Taggart, P. M. Sutton, T. Opthof, R. Coronel, R. Trimlett, W. Pugsley, and P. Kallis. Inhomogeneous transmural conduction during early ischaemia in patients with coronary artery disease. J. Mol. Cell Cardiol., 32(4):621-630, April 2000.

[49] K.H. Ten Tusscher, R. Hren, and A.V. Panfilov. Organization of ventricular fibrillation in the human heart. Circ. Res., 100(12):e87-101, 2007.

[50] K.H. Ten Tusscher and A.V. Panfilov. Alternans and spiral breakup in a human ventricular tissue model. Am. J. Physiol. Heart Circ. Physiol., 291(3):H1088-100, 2006.

[51] K.H.W.J. Ten Tusscher, D. Noble, P.J. Noble, and A.V. Panfilov. A model for human ventricular tissue. Am. J. Physiol. Heart Circ. Physiol., 286:H1573-H1589, 2004.

[52] M. Valderrabano, P. Chen, and S.F. Lin. Spatial distribution of phase singularities in ventricular fibrillation. Circulation, 108:354-359, 2003.

[53] M. Vaquero, D. Calvo, and J. Jalife. Cardiac fibrillation: from ion channels to rotors in the human heart. Heart. Rhythm., 5(6):872-879, June 2008.

[54] J.N. Weiss, A. Garfinkel, H.S. Karagueuzian, Z. Qu, and P.S. Chen. Chaos and the transition to ventricular fibrillation: a new approach to antiarrhythmic drug evaluation. Circulation., 99(21):2819-2826, 1999.

[55] J.N. Weiss, A. Karma, Y. Shiferaw, P.S. Chen, A. Garfinkel, and Z. Qu. From pulsus to pulseless: the saga of cardiac alternans. Circ. Res., 98(10):1244-53, 2006.

[56] F.X. Witkowski, L.J. Leon, P.A. Penkoske, W.R. Giles, M.L. Spano, E.L. Ditto, and A.T. Winfree. Spatiotemporal evolution of ventricular fibrillation. Nature, 392:78-82, 1998.

[57] T. J. Wu, S. F. Lin, A. Baher, Z. Qu, A. Garfinkel, J. N. Weiss, C. T. Ting, and P. S. Chen. Mother rotors and the mechanisms of D600-induced type 2 ventricular fibrillation. Circulation., 110(15):2110-2118, October 2004.

[58] T. J. Wu, J. J. Ong, C. Hwang, J. J. Lee, M. C. Fishbein, L. Czer, A. Trento, C. Blanche, R. M. Kass, W. J. Mandel, H. S. Karagueuzian, and P. S. Chen. Characteristics of wave fronts during ventricular fibrillation in human hearts with dilated cardiomyopathy: role of increased fibrosis in the generation of reentry. J. Am. Coll. Cardiol., 32(1):187-196, July 1998.

[59] T.J. Wu, J.J.C. Ong, C. Hwang, J.J. Lee, M.C. Fishbein, L. Czer, A. Tento, C. Blanche, R.M. Kass, W.J. Mandel, H.S. Karagueuzuan, and P. Chen. Characteristics of wave fronts during ventricular fibrillation in human hearts with dilated cardiomyopathy: Role of increased fibrosis on the generation of reentry. J. Am. Coll. Cardiol., 32:187-196, 1998.

[60] A.M. Yue, M.R. Franz, P.R. Roberts, and J.M. Morgan. Global endocardial electrical restitution in human right and left ventricles determined by noncontact mapping. J. Am. Coll. Cardiol., 46(6):1067-75, 2005.

[61] A. V. Zaitsev, O. Berenfeld, S. F. Mironov, J. Jalife, and A. M. Pertsov. Distribution of excitation frequencies on the epicardial and endocardial surfaces of fibrillating ventricular wall of the sheep heart. Circ. Res., 86(4):408-417, March 2000.

[62] M.E. Zevitz. Ventricular fibrillation [online]. eMedicine, http://www.emedicine.com/med/topic2363.htm, 2004. 


\section{Figure Legends}

\section{Figure 1}

3D plots of maximum restitution curves $S_{\max }$. From left to right: $L V, R V$, anterior and posterior views. The circles denote the positions of initial wave breaks leading to the formation of the first spiral wave. Black circles indicate positions of the first wavebreak that lead to multiple wavelet VF dynamics, whereas white circles indicate positions of first wavebreak that lead to mother rotor fibrillation type dynamics. Maximum restitution slope values range from 0.5 (blue) to 3 (red).

\section{Figure 2}

Multiple wavelet VF. Wavefronts are shown in red. Top: LV view. Bottom: RV view. First column: location of the initial spiral, second column: after 5 seconds, third column: after 7 seconds. Most wave breaks occur in the RV free wall and exist independently.

\section{Figure 3}

Mother rotor fibrillation. Wavefronts are shown in red. Top: LV view. Bottom: RV view. First column: location of the initial spiral, second column: after 3.5 seconds, third column: after 10 seconds. Note the stable spiral in the LV free wall, while breaks occurred in the RV free wall. The spiral in the LV wall remained stable until the end of the simulation (up to 12 seconds simulated). Breaks in the RV were driven by the mother rotor spiral.

\section{Figure 4}

Ablation of the mother rotor spiral in the LV (posterior view). The black dashed line denotes the part of the ventricles that was removed. The first snapshot is at approximately 3 seconds of simulation time (i.e., the time when part of the LV was removed), the second snapshot at 3.3 seconds and the third snapshot is at 3.5 seconds, after which there was no longer any wave activity.

\section{Figure 5}

ECG $(A, B)$. Histogram of the mean period distribution measured in all heart points between 2-6 seconds of simulation time (C,D). Left: multiple wavelet simulation. Right: mother rotor simulation.

\section{Figure 6}

Normalized period distributions. Local mother rotor properties are changed ( $\mathrm{I}_{\mathrm{CaL}}$ times 1.0, 0.5, 0.35 and 0.25.).The gray vertical line denotes the mean period value of $\mathrm{I}_{\mathrm{CaL}}$ times 1.0 (panel A).

\section{Figure 7}

Spatial period distribution for the simulation with $\mathrm{I}_{\mathrm{CaL}}$ times 0.25 . A) 2-4 seconds, B) 4-6 seconds. The mother rotor was located in the dark blue region. Period values range from $0.18 \mathrm{~s}$ (blue) to $0.28 \mathrm{~s}$ (red).

\section{Tables}

\section{Table 1}

\begin{tabular}{|l|l|l|l|}
\hline init & type & \#filaments & avg. lifespan (sec) \\
\hline LV & MR & 388 & 0.068 \\
LV-ANT & MR & 518 & 0.061 \\
\hline LV-POST & MW & 1268 & 0.081 \\
POST & MW & 1025 & 0.084 \\
RV-POST & MW & 952 & 0.082 \\
RV & MW & 978 & 0.079 \\
SEP & MW & 980 & 0.084 \\
\hline
\end{tabular}

Table 1: Total number of filaments detected during 8 seconds of simulation time for different locations of the initial scroll wave (init). LV: left ventricle. RV: right ventricle. POST: posterior. ANT: anterior. SEP: septum. MR: mother rotor fibrillation, MW: multiple wavelet VF. 

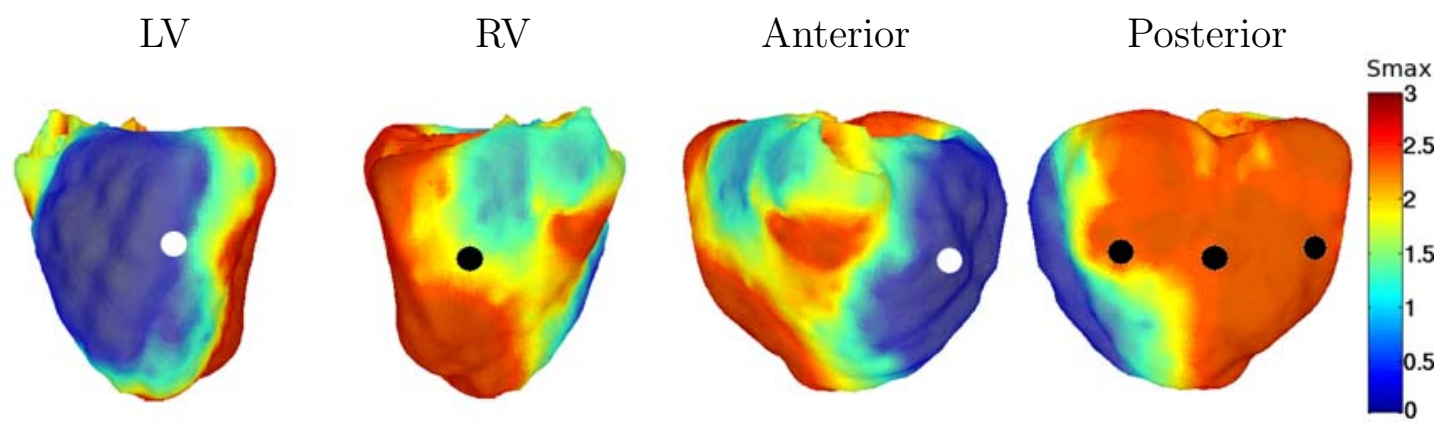
㽞 
㬝 

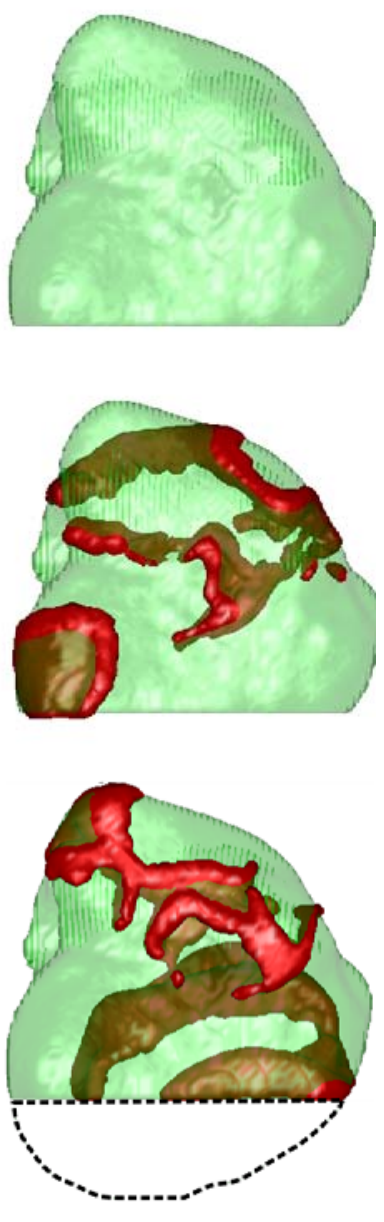
multiple wavelet
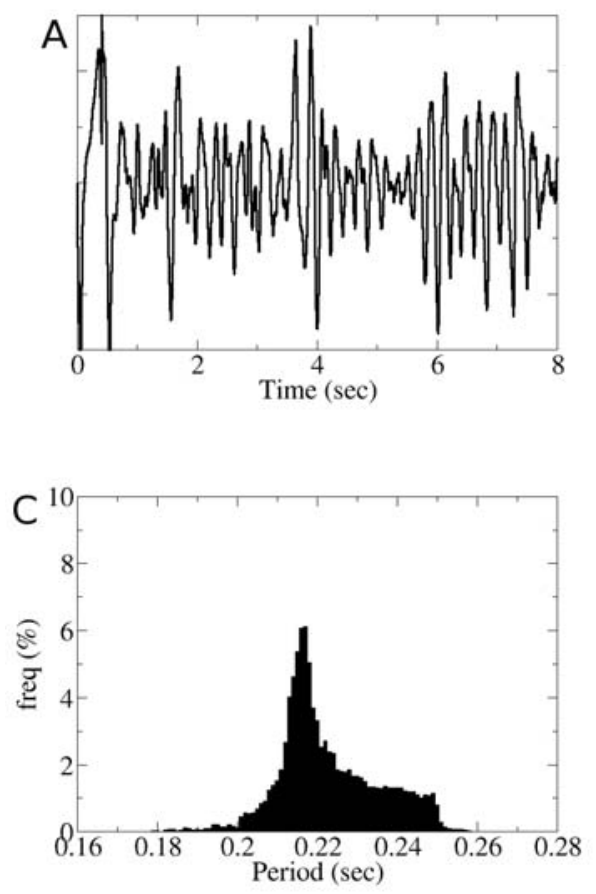

mother rotor
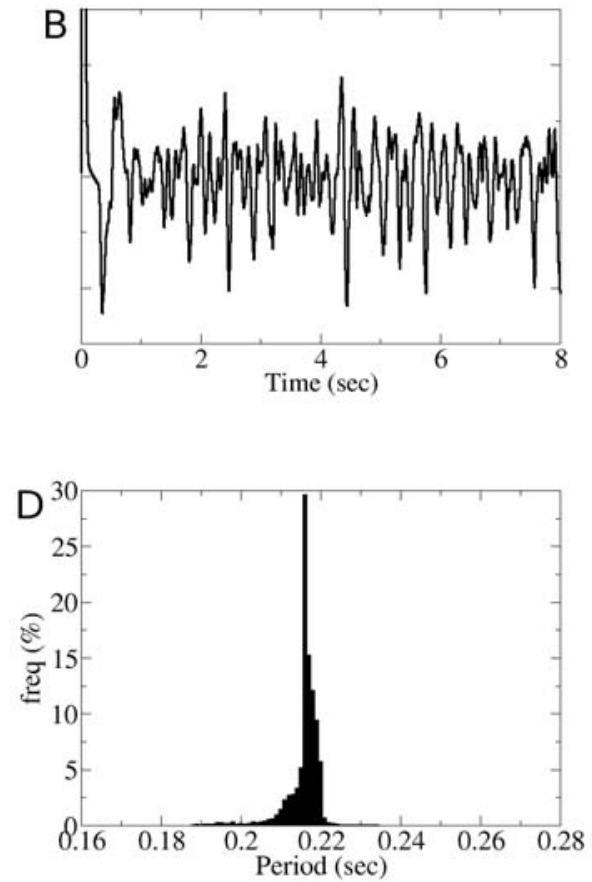

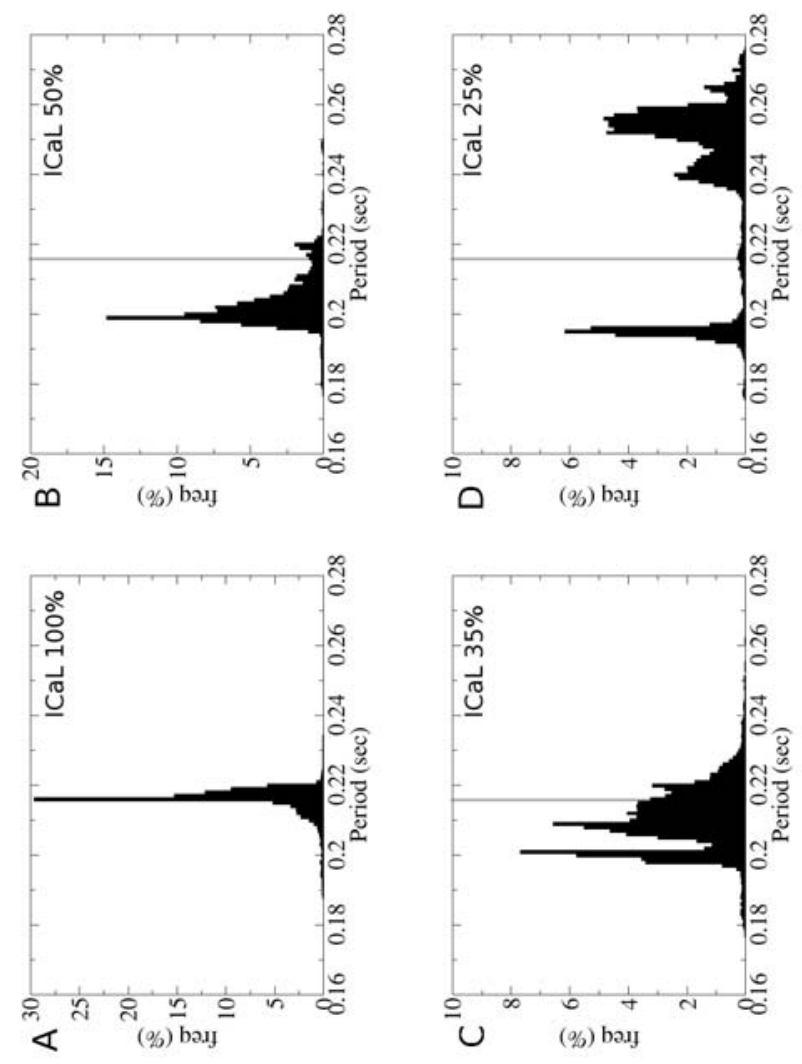


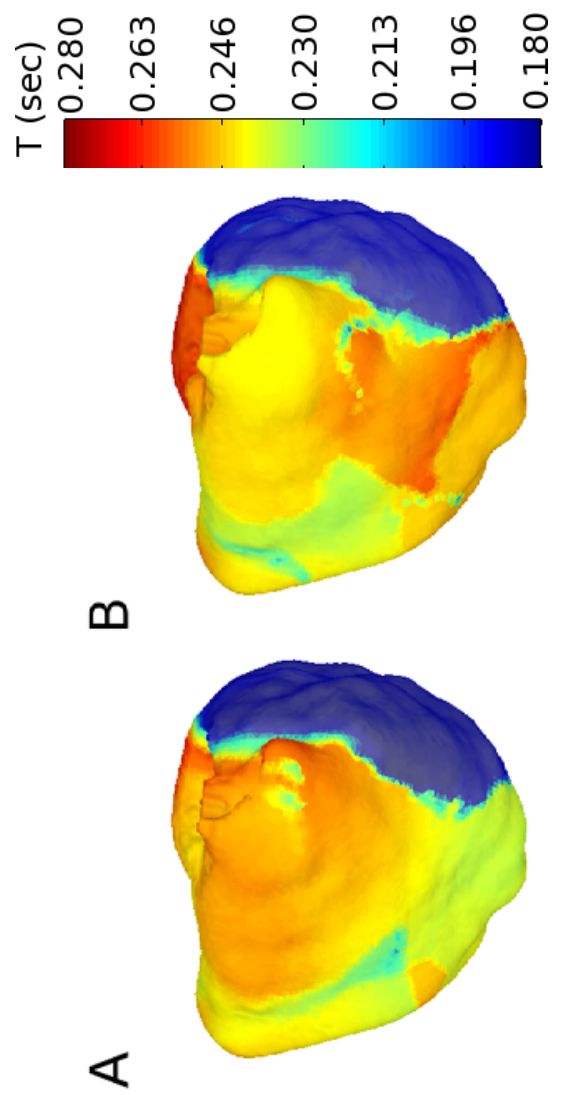

